\title{
Do padrão nightingaleano de enfermagem ao sistema social luhmanniano: estudo teórico
}

\author{
From nursing nightingalean pattern to Luhmann's social system: theoretical study
}

Del patrón nightingaleano de enfermería al sistema social de Luhmann: estudio teórico

\author{
Dirce Stein Backes', Marli Terezinha Stein Backes", Alacoque Lorenzini Erdmann"I", Andreas Büscher ${ }^{\mathrm{IV}}$ \\ 'Centro Universitário Franciscano, Curso de Enfermagem. Santa Maria-RS, Brasil. Universidade Federal do Rio Grande, \\ Programa de Pós-Graduação de Enfermagem. Rio Grande-RS, Brasil. \\ "Universidade Federal de Pelotas, Hospital Escola da UFPel. Pelotas-RS, Brasil. \\ I" Universidade Federal de Santa Catarina, Centro de Ciências da Saúde, Departamento de Enfermagem, \\ Programa de Pós-Graduação em Enfermagem. Florianópolis-SC, Brasil. \\ IV Fakultät Wirtschafts und Sozialwissenschaften, Deutsches Netzwerk für Qualitätsentwicklung in der Pflege (DNQP), \\ Osnabrück, Germany.
}

Submissão: 20-04-2013 Aprovação: 12-06-2013

RESUMO

O presente estudo, de caráter teórico, objetivou analisar e discutir um possível código binário para o sistema de Enfermagem, no sentido de identificar o seu próprio saber, na perspectiva dos pressupostos teóricos de Niklas Luhmann. Apostar em uma comunicação funcionalmente diferenciada e socialmente relevante para o sistema de enfermagem implica em transcender o tradicional código saúde-doença, predominante no sistema de saúde e cuja comunicação socialmente relevante é a doença. Implica, ainda, em investir proativamente na promoção e proteção do viver saudável de indivíduos, famílias e comunidades, para que a saúde seja o ponto central das discussões e intervenções.

Descritores: Cuidados de Enfermagem; Papel do Profissional de Enfermagem; Dinâmica não Linear; Teoria de Sistemas.

\section{ABSTRACT}

The purpose of this study, of a theoretical nature, was to analyze and discuss a possible binary code for the Nursing system, in the sense of identifying its own knowledge from the perspective of Niklas Luhmann's theoretical presuppositions. To bet on functionally differentiated and socially relevant communication for the nursing system implies transcending the traditional health-disease code, predominant in the health system, whose socially relevant communication is the disease. Furthermore, it implies in investing proactively in the promotion and protection of healthy living of individuals, families and communities, so that health will be the central point of discussions and interventions.

Key words: Nursing Care; Nurse's Role; Nonlinear Dynamics; Systems Theory.

\section{RESUMEN}

El presente estudio de carácter teórico objetivó analizar y discutir un código binario para el sistema de la enfermería con el finalidad de identificar sus propios conocimientos desde la perspectiva de los supuestos teóricos de Niklas Luhmann. La apuesta en una comunicación funcionalmente diferenciada y socialmente relevante para el sistema de enfermería, significa transcender el código tradicional de la salud y la enfermedad, predominante en el sistema de la salud y la divulgación de las cuales es la enfermedad de relevancia social. También implica investir, de manera proactiva, en la promoción y la protección de la vida saludable de los individuos, familias y comunidades para asegurar que la salud sea el punto central de los debates e intervenciones.

Palabras clave: Atención de Enfermería; Rol de la Enfermera; Dinámica no Lineal; Teoría de sistemas.

\section{AUTOR CORRESPONDENTE}

Dirce Stein Backes

E-mail: backesdirce@ig.com.br 


\section{INTRODUÇÃO}

O advento da Enfermagem, no Brasil, representou um importante marco na mobilização e trasformação das questões de saúde. A mesma surgiu, em um momento em que o Estado brasileiro instituíra políticas de saúde voltadas para o controle das endemias e das epidemias, as quais colocavam o Brasil numa posição ameaçadora ao desenvolvimento do comércio internacional ${ }^{(1)}$.

Ao tomar conhecimento do padrão nightingaleano das enfermeiras norte-americanas, o então diretor da Cruz Vermelha acreditou ser este o profissional necessário para a estratégia sanitarista do governo brasileiro e solicitou a criação de serviço semelhante, no Brasil. Criada, no ano de 1922, com base no "padrão Nightingaleano", a Escola de Enfermagem do Departamento Nacional de Saúde Pública do Rio de Janeiro orientou-se no modelo de saúde vigente, o qual estava centrado em abordagens biomédico-curativistas e, consequentemente, com foco na doença ${ }^{(2-3)}$.

Com tal enfoque, o profissional de enfermagem, como também os demais profissionais de saúde, pautaram o seu saber técnico-científico em práticas biologicistas e assistencialistas, nas quais predominavam o enfoque da doença, da cura e da manutenção do status quo ${ }^{(4)}$.

Nesse processo histórico, a construção do conhecimento na área de Enfermagem vem se dando em fases evolutivas, as quais não se sucedem de forma linear ou pontual, mas processual e complementar ${ }^{(5)}$. No período de Florence Nightingale, primeira fase, o foco do saber de Enfermagem centrou-se em "o que fazer?" Na segunda, tentando conquistar o domínio técnico, a Enfermagem procurou definir "como fazer?" Na terceira, focou-se em princípios científicos e investigou "por que fazer?" Atualmente, busca discutir "qual o saber próprio da enfermagem?".

O último questionamento se justifica, no cenário brasileiro, pela necessidade de repensar o modelo de atenção à saúde e desenvolver saberes que contribuam para a consolidação do Sistema Único de Saúde brasileiro (SUS). Com foco na prevenção, promoção e proteção à saúde, o SUS transcende o modelo biomédico-curativista com foco na doença e visa alcançar uma concepção sistêmica em saúde, no sentido de potencializar o viver saudável de indivíduos, famílias e comunidades.

Com base no exposto, este estudo, de caráter teórico, objetivou analisar e discutir um possível código binário para o sistema de Enfermagem, no sentido de identificar o seu próprio saber, na perspectiva dos pressupostos teóricos de Niklas Luhmann.

\section{SISTEMAS FUNCIONALMENTE DIFERENCIADOS}

Na perspectiva luhmanniana, um sistema se diferencia não pela posição estrutural ou hierárquica que ocupa, mas pela função social que desempenha a partir de um código de diferenciação funcional. O sistema se caracteriza pela garantia da auto-referência, isto é, pela capacidade de identificar e assegurar o seu próprio saber, nesse caso, o saber da Enfermagem ${ }^{(6-8)}$.

Para que um sistema se diferencie funcionalmente ele necessita, na concepção luhmanniana, garantir o seu próprio saber mediante um código binário, isto é, mediante um código de distinção de duas possibilidades comunicativas ${ }^{(9-13)}$. No sistema de saúde, por exemplo, a comunicação de sentido se estabelece por meio do código de diferenciação saúde-doença. Nessa relação, a doença é considerada, frequentemente, o ponto positivo e a saúde o ponto negativo do processo de diferenciação funcional. Logo, o saber da medicina como o saber dos demais profissionais de saúde é orientado pelo modelo biomédico-curativista, cuja ênfase reside na "doença do indivíduo" e a comunicação socialmente relevante é reduzida à doença $a^{(12-13)}$.

Para ser considerado um sistema funcionalmente diferenciado e assegurar o seu próprio saber, a Enfermagem precisa desenvolver, a exemplo de outros sistemas sociais, a sua própria comunicação de sentido, mediante um código binário específico $^{(11)}$. Esse código de diferenciação funcional deve ser garantido, no entanto, a partir de uma circularidade interativa com os demais subsistemas de saúde, visto que na concepção sistêmica não existem relações hierárquicas, mas interações circulares e dialógicas.

Nessa direção, questiona-se: é possível desenvolver um código binário específico para o sistema de enfermagem, capaz de transcender o tradicional código saúde-doença predominante no sistema de saúde, cuja comunicação socialmente relevante é a doença? Quais novos saberes é preciso analisar e discutir para que a Enfermagem se constitua em um sistema funcionalmente diferenciado?

\section{SISTEMA DE ENFERMAGEM NA PERSPECTIVA DE PES- QUISADORES ALEMÃES}

Já existem razões para se pensar em um código binário específico para o sistema de Enfermagem. Pesquisadores alemães, a partir de estudos fundamentados na teoria dos sistemas sociais de Niklas Luhmann, discutem possíveis códigos que podem ser considerados pela Enfermagem ${ }^{(9,11,13)}$. Um deles diz respeito ao "cuidado-descuidado", o outro se refere à "necessidade de cuidado-não necessidade de cuidado" e um terceiro, considera a "competência para o cuidado-não competência para o cuidado". Sem chegar a definições conclusivas, as discussões convergem para o código "competência para o cuidado-não competência para o cuidado", visto que tal diferenciação funcional possibilita ao sistema assegurar o seu próprio saber, sem excluir as demais possibilidades interativas e associativas.

Para Luhmann, é somente mediante um código específico que se pode pensar em sistemas auto-referenciados e autônomos. Os sistemas autônomos, na compreensão luhmanniana, não se apóiam em regras morais, religiosas, civis ou políticas, mas se identificam e diferenciam pela função social que desempenham ${ }^{(9)}$. Sob esse enfoque, o sistema de Enfermagem precisa, crescentemente, ampliar e definir melhor o objeto de sua práxis social, isto é, a comunicação socialmente relevante, a fim de promover e assegurar a sua autonomia.

Para os sociólogos alemães, a Enfermagem é uma prática social por excelência. Uma prática, todavia, que se desenvolveu com base no tradicional e hegemônico código saúde-doença e, consequentemente, a partir de relações hierárquicas, nas quais prevalece o saber médico. Desenvolveu-se, 
portanto, com base em práticas assistencialistas e num contexto em que a "doença" predominou como comunicação socialmente relevante ${ }^{(13)}$.

Para assegurar o saber autônomo ou funcionalmente diferenciado, o sistema de enfermagem precisa, nessa relação, transcender os reducionismos provocados pelo código saúde-doença, cuja comunicação socialmente relevante é a doença. Para estar em consonância com a atual política brasileira de saúde - SUS, o sistema de Enfermagem necessita ampliar o seu campo de visão, pela compreensão do cuidado em saúde como fenômeno complexo, sistematizado por meio das múltiplas relações, interações e associações sistêmicas, com vistas a promover a saúde do ser humano de forma integral e articulada com tudo que o cerca.

\section{CÓDIGO DE DIFERENCIAÇÃO FUNCIONAL PARA O SIS- TEMA DE ENFERMAGEM}

A concepção que fundamenta o SUS e a Estratégia Saúde da Família conferiu à Enfermagem Brasileira, pela compreensão ampliada do processo saúde-doença, um importante espaço de inserção social e uma identidade funcionalmente diferenciada. Essa identidade funcional necessita, no entanto, de níveis teóricos mais avançados, no sentido de alcançar um pensamento complexo - sistêmico acerca do saber próprio da Enfermagem.

Uma importante discussão teórica está associada ao cuidado pontual $X$ cuidado ampliado e/ou ao cuidado da parte $X$ cuidado do todo, cujo binômio vem sendo evidenciado como um possível código de diferenciação funcional para o sistema de Enfermagem, do Brasil. Uma visão contextualizada e sistêmica permite argumentar que, enquanto os profissionais de saúde, de modo geral, buscam atender uma parte específica do ser humano, isto é, a parte biológica, a parte nutricional, a parte ocupacional, a parte mental, o fígado doente e outros, o profissional de Enfermagem tem a possibilidade de alcançar o todo do ser humano, pela integralidade do cuidado. A Enfermagem tem a oportunidade de promover o cuidado ampliado, a partir da compreensão do ser humano como unidade complexa e do cuidado de enfermagem como fenômeno complexo-sistêmico.

Pela compreensão circular e interativa do cuidado, a Enfermagem tem a possibilidade de promover a interconexão das partes com o todo, das partes entre as partes e do todo com as partes, no sentido de apreender o ser humano como um ser singular e multidimensional. Nesse processo, não raramente, a Enfermagem se identifica como a mola propulsora e integradora das partes ao todo e do todo às partes, a fim de atingir a unidade complexa - ser humano como ser singular e multidimensional.

O entendimento de complexidade, onde não apenas a parte está no todo, como o todo está inscrito na parte, traduz a conexão entre a unidade e a multiplicidade, "do que foi tecido junto" (14), isto é, quando elementos "diferentes são inseparáveis, constitutivos do todo e há um tecido (cuidado) interdependente, interativo e circular entre o objeto do conhecimento e o seu contexto, as partes e o todo, o todo e as partes e as partes entre si. Nessa relação, o todo (cuidado) é uma unidade complexa que não se reduz à soma dos elementos que constituem as partes. Considera-se, portanto, que a soma das partes é maior e menor que o todo, considerando que cada parte apresenta as suas especificidades e, na interação umas com as outras, modificam-se as partes e também o todo para apreender o ser humano como singular e multidimensional ${ }^{(14)}$.

Ao mesmo tempo em que se evidencia como possível código de diferenciação funcional da Enfermagem o "cuidado da parte-cuidado do todo", se observa, na mesma dimensão, a fragmentação, a dissociação e a descontinuidade do cuidado em saúde. Evidencia-se, que o ser humano, frequentemente, é apreendido como resultado da soma matemática das partes ou como um todo divisível, isto é, dividido em partes, no qual cada parte se constitui em fragmento para determinada especialidade. Sob esse direcionamento, questiona-se: A quem (profissional) cabe a função de interconectar as partes para formar a unidade complexa?

Reconhece-se, que o campo profissional mais preparado e habilitado para interconectar as partes ou os diferentes "fios interativos" do cuidado em saúde é a Enfermagem. Em sua formação profissional, o Enfermeiro desenvolve uma "visão global e sistêmica, pautada em saberes técnico-científicos e humanos para atuar de forma crítico-reflexiva e proativa nos diferentes cenários de atenção à saúde"(15).

Tais saberes globais e sistêmicos conferem à Enfermagem a liderança pelo processo de cuidado como um todo. Da mesma forma, possibilitam a função de organizar o ambiente de cuidado para os demais profissionais de saúde, promover a interlocução entre o paciente, a família, a equipe de saúde e a comunidade. A Enfermagem constitui-se, portanto, em mola propulsora e dinamizadora do cuidado sistêmico, no qual e para o qual as diferentes partes são inevitáveis para a compreensão do todo - unidade complexa. Nessa circularidade interativa, a Enfermagem organiza, sistematiza, dinamiza e lidera o cuidado complexo, como processo de múltiplas relações, interações e associações.

Figura 1 - Enfermagem como mola propulsora do cuidado sistêmico

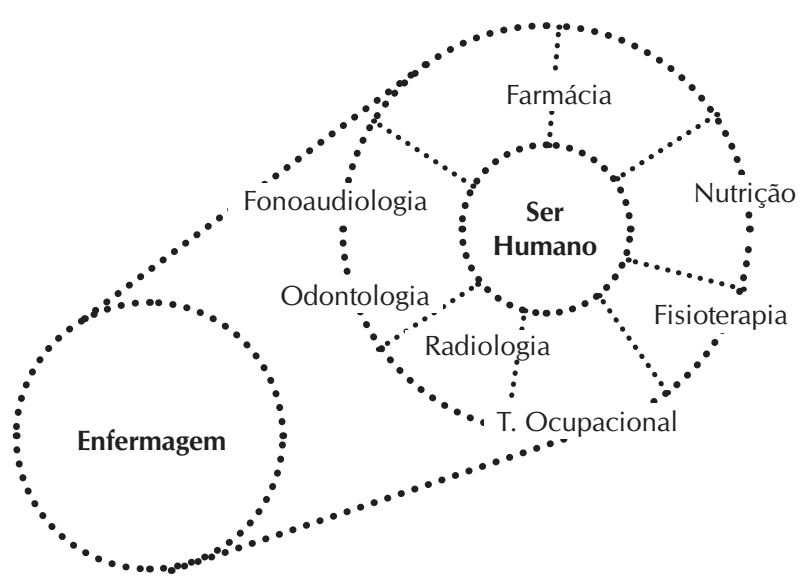

O cuidado sistêmico, entendido a partir "do que foi tecido junto" para formar a unidade complexa, não tem a pretensão de excluir as partes ou reduzir o cuidado a uma ação pontual de Enfermagem. Essa forma de pensar, não passaria de uma nova concepção reducionista, considerando que o sistema de 
Enfermagem desenvolve a sua auto-referência e autonomia funcional a partir de uma relação de interdependência com demais sistemas funcionalmente diferenciados ${ }^{(6)}$.

Nessa direção, a relevância do sistema de Enfermagem não está na posição estrutural ou hierárquica que ocupa, mas na capacidade de estabelecer relações e associações de interdependência com os demais subsistemas de saúde. Assim, o sistema de Enfermagem será identificado e concebido não apenas pelo cuidado linear e pontual, mas pela capacidade de desenvolver novas e sempre mais complexas comunicações intersistêmicas. Para tanto, requer cada vez mais, o desenvolvimento de saberes interativos e associativos, no sentido de fomentar e liderar o cuidado como unidade complexa e sistêmica.

Entende-se, que o pensamento complexo-sistêmico aspira ao conhecimento singular e multidimensional. Logo, comporta um princípio de incompletude, interdependência e complementaridade $^{(16)}$. Aderir a esse pensamento significa considerar os múltiplos "fios" que interagem e se complementam para formar o cuidado complexo-sistêmico em saúde, com vistas a compreensão do ser humano como unidade complexa.

\section{CONSIDERAÇÕES FINAIS}

São inegáveis os avanços da Enfermagem brasileira na busca de sua identidade e autonomia profissional. Permanecem, no entanto, como desafios, na luta pelo reconhecimento da profissão de Enfermagem como sistema funcionalmente diferenciado: a necessidade de fundamentar o saber técnico-científico com base em referenciais que ampliem o conhecimento próprio de Enfermagem como fenômeno complexo e a adoção de estratégias que permitam legitimar e incorporar o conhecimento científico produzido ao campo de Enfermagem.

Para o sistema de Enfermagem transcender o tradicional código saúde-doença, cuja comunicação funcionalmente diferenciada e socialmente relevante é a doença, o mesmo necessita apostar em uma comunicação funcionalmente diferenciada e socialmente relevante. Implica, ainda, em investir proativamente na promoção e proteção do viver saudável de indivíduos, famílias e comunidades, para que a saúde se torne o ponto central das discussões e intervenções em saúde. Nessa direção, o empreendedorismo e as redes interativas e associativas se constituem em importantes mecanismos mobilizadores em vista da conquista de novos espaços e de novas possibilidades de intervenção social.

A análise e as discussões acerca de um possível código binário para o sistema de Enfermagem suscitam novas questões e reflexões, entre as quais: Que saberes são necessários no processo de formação profissional e atuação do Enfermeiro, para que seja capaz de fomentar processos interativos, associativos e de interdependência com os demais campos profissionais? Que novos saberes necessitam ser construídos, a fim de conceber o cuidado de Enfermagem como unidade complexa?

\section{REFERÊNCIAS}

1. Silva AL. O saber nightingaliano no cuidado: uma abordagem epistemológica. In: Lopes MJM, Meyer DE, Waldow VR. Maneiras de cuidar, maneiras de ensinar: a enfermagem entre a escola e a prática profissional. Porto Alegre: Artes Médicas; 1995.

2. Paixão W. História da Enfermagem. 5. ed. Rio de Janeiro: Júlio C. Reis; 1979.

3. Geovanini T. História da Enfermagem: versões e interpretações. Rio de Janeiro: Livraria Editora Revinter; 1995.

4. Backes DS, Backes MS, Erdmann AL. A prática social sistêmica do enfermeiro na perspectiva luhmanniana. Rev Esc Enferm USP 201;45(1):116-121.

5. Gomes VLO, Backes VMS, Padilha MICS, Vaz MRC. Evolução do conhecimento científico na enfermagem: do cuidado popular à construção de teorias. Invest Educ Enferm 2007;25(2):108-115.

6. Luhmann N. Soziale Systeme: Grundriß einer allgemeinen theorie. Frankfurt: Main; 1984.

7. Luhmann N. Sistemas Sociales. Lineamentos para una teoría general. Barcelona: Anthropos, México: Universidade Iberoamericana, Santafé de Bogotá: CEJA; 1998.

8. Luhmann N. Der medizinische Code. In: Luhmann N. Soziologische Aufklärung 5: Konstrutivistische Perspektiven. Opladen: Westdeutscher Verlang; 1990.

9. Hohm HJ. Das Pflegesystem. Seine Organisationen und
Karrieren. Systemtheoretische Beobachtungen zur Entstehung eines sekundären Kunktionssystems. Freiburg: Lambertus; 2002 .

10. Schroeter KR, Rosenthal T. Soziologie der Pflege: Grundlagen, Wissensbestände und Perspektiven. Weinheim, München: Juventa; 2005.

11. Schroeter KR. Das soziale Feld der Pflege: Eine Einführung in Strukturen, Deutungen und Handlungen. München: Juventa; 2006.

12. Bauch J. Pflege als soziales System. In: Schoroter KR, Rosenthal T. Soziologie der Pflege. Grundlagen, Wissensbestände und Perspektiven. Weinheim, München: Juventa; 2005.

13. Bauch J, Fuchs P, Hafen M, Kopfdquter K, Kraft V, Krause $\mathrm{T}$, et al. Pflege als soziales System. In: Bauch J. (Hrsg). Gesundheit als System: Systemtheoretische Beobachtungen des Gesundheitswesens. Hartung-Gorre Verlang Konstanz; 2006.

14. Morin E. Os sete saberes necessários a educação do futuro. 6. ed. São Paulo: Cortez; 2002.

15. Backes DS. Empreendedorismo social da enfermagem: rupturas e avanços. Santa Maria: Centro Universitário Franciscano; 2012.

16. Morin E. Introdução ao pensamento complexo. 5. ed. Lisboa: Instituto Piaget; 2012. 